\title{
A partial generalization of the Livingstone-Wagner Theorem
}

\author{
Yasuhiro Nakashima \\ Graduate School of Information Sciences \\ Tohoku University, Japan
}

Received 22 October 2008, accepted 1 November 2009, published online 5 November 2009

\begin{abstract}
For a transitive permutation group $G$ on a finite set $\Omega$, the Livingstone-Wagner Theorem states that if $G$ is $k$-homogeneous and $2 \leq k \leq \frac{|\Omega|}{2}$, then $G$ is $(k-1)$-transitive. We conjecture that the number of $G$-orbits on $k$-subsets of $\Omega$ is greater than or equal to the number of $G$-orbits on ordered $(k-1)$-tuples of $\Omega$, if $|\Omega|$ is sufficiently large. For the simplest case $k=3$, we verify this conjecture by establishing a result on edge-colorings of complete digraphs.
\end{abstract}

Keywords: Groups, transitivity, orbits.

Math. Subj. Class.: 20B20, 20B35

\section{Introduction}

Let $G$ be a permutation group on a finite set $\Omega$. Denote by $\Omega / G$ the set of $G$-orbits on $\Omega$. Let $\Omega_{(k)}$ and $\Omega_{[l]}$ denote the family of all $k$-subsets of $\Omega$ and the family of all $l$-tuples of distinct elements of $\Omega$, respectively. The group $G$ is said to be $k$-homogeneous or $l$-transitive if $G$ acts transitively on $\Omega_{(k)}$ or $\Omega_{[l]}$ respectively.

Livingstone and Wagner [3, Theorem 1,2] showed that for any group $G$ acting on $\Omega$, and for any $k$ with $2 \leq k \leq \frac{|\Omega|}{2}$,

(1) the inequality $\left|\Omega_{(k)} / G\right| \geq\left|\Omega_{(k-1)} / G\right|$ holds. In particular, $k$-homogeneity implies also $(k-1)$-homogeneity,

(2) if $G$ is $k$-homogeneous, then $G$ is $(k-1)$-transitive, and

(3) for $k \geq 5$, if $G$ is $k$-homogeneous, then $G$ is $k$-transitive.

E-mail address: ysnakashima@gmail.com (Yasuhiro Nakashima) 
Martin and Sagan [4, Theorem 2] generalized (1) by introducing the concept of $\lambda$ transitivity as follows. Let $S_{\lambda}$ be the set of all partitions of $\Omega$ of shape $\lambda$. If a permutation group $G$ on $\Omega$ acts transitively on $S_{\lambda}$, then $G$ is said to be $\lambda$-transitive. Let $\unlhd$ denote the dominance order (see [5, Definition 2.2.2]) on the set of partitions of $|\Omega|$. Martin and Sagan proved that $\lambda \unlhd \mu$ implies $\left|S_{\lambda} / G\right| \geq\left|S_{\mu} / G\right|$. In particular, $\lambda$-transitivity implies $\mu$-transitivity. Since $S_{(|\Omega|-k, k)} \cong \Omega_{(k)}$ and $S_{(|\Omega|-k+1, k-1)} \cong \Omega_{(k-1)}$ as $G$-sets, the Livingstone-Wagner Theorem (1) follows from the Martin-Sagan Theorem. Analogously, (2) will follow if we could prove $\left|\Omega_{(k)} / G\right| \geq\left|\Omega_{[k-1]} / G\right|$. Since $\left|\Omega_{(k)}\right| \geq\left|\Omega_{[k-1]}\right|$ holds when $|\Omega| \geq k !+k-1$, one may expect the inequality $\left|\Omega_{(k)} / G\right| \geq\left|\Omega_{[k-1]} / G\right|$ to be true for $|\Omega|$ sufficiently large. Since $S_{\left(|\Omega|-k+1,1^{k-1}\right)} \cong \Omega_{[k-1]}$ as $G$-sets, and the partitions $(|\Omega|-k, k),\left(|\Omega|-k+1,1^{k-1}\right)$ are incomparable with respect to the dominance order, Theorem 2 of [4] does not apply. For the simplest case $k=3$, the inequality follows from the following result of Cameron and Saxl [1], provided that $\left|\Omega_{[2]} / G\right| \geq 26$.

Proposition 1.1 ([1]). Let $G$ be a transitive permutation group on a set $\Omega$ with $|\Omega|>2$. Then $\left|\Omega_{(3)} / G\right| \geq \frac{\left|\Omega_{(2)} / G\right|\left(\left|\Omega_{(2)} / G\right|-1\right)}{6}$.

Indeed, since $2\left|\Omega_{(2)} / G\right| \geq\left|\Omega_{[2]} / G\right|$, Proposition 1.1 implies

$$
\left|\Omega_{(3)} / G\right| \geq \frac{\left|\Omega_{[2]} / G\right|\left(\left|\Omega_{[2]} / G\right|-2\right)}{24} \geq\left|\Omega_{[2]} / G\right|
$$

provided that $\left|\Omega_{[2]} / G\right| \geq 26$.

The purpose of this paper is to prove the inequality $\left|\Omega_{(3)} / G\right| \geq\left|\Omega_{[2]} / G\right|$ provided that $|\Omega| \geq 11$, by counting certain configurations in a regular edge-coloring of a complete digraph (the definition of which is given in the next section). Here we only note that every transitive permutation group $G$ on $\Omega$ induces a regular edge-coloring $\left(\Omega, \mathcal{C}_{G}, \phi_{G}, \Psi_{G}\right)$ with $\mathcal{C}_{G}=\Omega_{[2]} / G$, and the number $\left|\overline{\Omega_{3}}\right|$ of equivalence classes is at most $\left|\Omega_{(3)} / G\right|$. We prove in Section 5 that if $(\Omega, \mathcal{C}, \phi, \Psi)$ is a regular edge-coloring with $|\Omega| \geq 11$, then $\left|\overline{\Omega_{3}}\right| \geq|\mathcal{C}|$. As a corollary to this result, we obtain the following.

Theorem 1.2. For any transitive permutation group $G$ on $\Omega$ with $|\Omega| \geq 11,\left|\Omega_{(3)} / G\right| \geq$ $\left|\Omega_{[2]} / G\right|$ holds.

For some permutation groups of degree less than 11, Theorem 1.2 fails to hold. In fact, $C_{6}, C_{3} \rtimes S_{2}, C_{3} \nmid S_{2}$, of degree $6, C_{7}$ of degree $7, C_{4} 2 S_{2}$ of degree 8 , and $C_{5} 2 S_{2}$ of degree 10 are all the counterexamples with degree greater than 5 . We refer the reader to [2] for unexplained notation in permutation group theory.

\section{Regular edge-colorings}

Let $\Omega, \mathcal{C}$ be finite sets, and let $\phi: \Omega_{[2]} \rightarrow \mathcal{C}$ be a surjective mapping. We call $(\Omega, \mathcal{C}, \phi, \Psi)$ a regular edge-coloring if

(R1) For each $a \in \mathcal{C}$ and each $\alpha \in \Omega$, there exists a positive integer $\delta_{a}$ such that

$$
|\{\beta \in \Omega \mid \phi(\alpha, \beta)=a\}|=\delta_{a}
$$

(R2) There is a bijective mapping $\Psi: \mathcal{C} \rightarrow \mathcal{C}$, which maps a color of an edge to that of its opposite:

$$
\text { for all }(\alpha, \beta) \in \Omega_{[2]},(\Psi \circ \phi)(\alpha, \beta)=\phi(\beta, \alpha) \text {. }
$$


Let $G$ be a transitive permutation group on $\Omega$. We obtain a regular edge-coloring induced by $G$, denoted $\left(\Omega, \mathcal{C}_{G}, \phi_{G}, \Psi_{G}\right)$, as follows. Let $\mathcal{C}_{G}=\Omega_{[2]} / G$, and define $\phi_{G}$ : $\Omega_{[2]} \rightarrow \mathcal{C}_{G}$ by $\phi_{G}(\alpha, \beta)=(\alpha, \beta)^{G}$ for $(\alpha, \beta) \in \Omega_{[2]}$, where $(\alpha, \beta)^{G}$ denotes the $G$-orbit of $(\alpha, \beta)$. Define $\Psi_{G}$ by $\Psi_{G}\left(\phi_{G}(\alpha, \beta)\right)=\phi_{G}(\beta, \alpha)$. Then (R1) holds by transitivity of $G$, and clearly (R2) holds. Thus $\left(\Omega, \mathcal{C}_{G}, \phi_{G}, \Psi_{G}\right)$ is a regular edge-coloring.

For the remainder of this section, we assume that a regular edge-coloring $(\Omega, \mathcal{C}, \phi, \Psi)$ is given. For $A, B \in \Omega_{(3)}$, we write $A \sim B$ if there exists a bijection $\pi$ from $A$ to $B$ such that $\phi\left(\pi(\alpha), \pi\left(\alpha^{\prime}\right)\right)=\phi\left(\alpha, \alpha^{\prime}\right)$ for any distinct $\alpha, \alpha^{\prime} \in A$. Then $\sim$ is an equivalence relation. Let $[A]$ denote the equivalence class of $A$. For $a, b, c \in \mathcal{C}$ we define

$$
[a, b, c]=\left\{\{\alpha, \beta, \gamma\} \in \Omega_{(3)} \mid \phi(\alpha, \beta)=a, \phi(\beta, \gamma)=b, \phi(\gamma, \alpha)=c\right\} .
$$

For each $A=\{\alpha, \beta, \gamma\} \in \Omega_{(3)}$, there exist $a, b, c \in \mathcal{C}$ such that $[A]=[a, b, c]$. Conversely, for any $a, b, c \in \mathcal{C}$, we can see that $[a, b, c]$ is an equivalence class unless it is empty. Let $\overline{\Omega_{3}}$ denote the set of equivalence classes with respect to $\sim$. For $a, b \in \mathcal{C}$, we define a family of equivalence classes $T_{a, b}$ by

$$
T_{a, b}=\{[a, b, x] \mid x \in \mathcal{C},[a, b, x] \neq \varnothing\} \subset \overline{\Omega_{3}} .
$$

By (R1), $T_{a, b}=\varnothing$ if and only if $b=\Psi(a)$ and $\delta_{a}=1$ for any $a, b \in \mathcal{C}, T_{c, c} \neq \varnothing$ for any $c \in \mathcal{C}$ with $c \neq \Psi(c)$, and $T_{\Psi(d), d} \neq \varnothing$ for any $d \in \mathcal{C}$ with $\delta_{d} \geq 2$. For convenience, we define $U_{a}, V_{a}$ for any $a \in \mathcal{C}$ by

$$
U_{a}=T_{\Psi(a), a}, \quad V_{a}=T_{a, a} .
$$

\section{Some lemmas}

Let $(\Omega, \mathcal{C}, \phi, \Psi)$ be a regular edge-coloring. We first prove some useful lemmas.

Lemma 3.1. Let $a, b, c, d, e \in \mathcal{C}$ with $[a, b, c] \neq \varnothing$. Then $[a, b, c] \in T_{d, e}$ if and only if $\{\Psi(d), e\} \in\{\{a, \Psi(c)\},\{b, \Psi(a)\},\{c, \Psi(b)\}\}$.

Proof. By the definition, the assertion follows from

$$
\begin{aligned}
& {[a, b, c] \in T_{d, e} } \\
\Longleftrightarrow & \text { there exists } x \in \mathcal{C} \text { such that }[a, b, c]=[d, e, x] \\
\Longleftrightarrow & (d, e) \in\{(a, b),(b, c),(c, a),(\Psi(a), \Psi(c)),(\Psi(b), \Psi(a)),(\Psi(c), \Psi(b))\} \\
\Longleftrightarrow & (\Psi(d), e) \in\{(\Psi(a), b),(\Psi(b), c),(\Psi(c), a),(a, \Psi(c)),(b, \Psi(a)),(c, \Psi(b))\} \\
\Longleftrightarrow & \{\Psi(d), e\} \in\{\{a, \Psi(c)\},\{b, \Psi(a)\},\{c, \Psi(b)\}\} .
\end{aligned}
$$

Lemma 3.2. Let $a, b, c, d$ be in $\mathcal{C}$ with $\{a, b\} \neq\{c, d\}$. If $\{\Psi(a), \Psi(b)\} \cap\{c, d\}=\varnothing$, then $T_{\Psi(a), b} \cap T_{\Psi(c), d}=\varnothing$.

Proof. Suppose $T_{\Psi(a), b} \cap T_{\Psi(c), d} \neq \varnothing$. Then there exists $x \in \mathcal{C}$ such that $[\Psi(a), b, x] \in$ $T_{\Psi(a), b},[\Psi(a), b, x] \neq \varnothing$ and $[\Psi(a), b, x] \in T_{\Psi(c), d}$. Lemma 3.1 implies that

$$
\{c, d\} \in\{\{\Psi(a), \Psi(x)\},\{b, a\},\{x, \Psi(b)\}\} .
$$

Thus $\{\Psi(a), \Psi(b)\} \cap\{c, d\} \neq \varnothing$, as $\{a, b\} \neq\{c, d\}$. 
Lemma 3.3. Let $a, b, c \in \mathcal{C}$ with $\Psi(a)=a$ and $b \notin\{c, \Psi(c)\}$. Then $T_{a, b} \cap T_{a, c}=$ $\{[a, b, \Psi(c)]\}$ if $[a, b, \Psi(c)] \neq \varnothing$, and $T_{a, b} \cap T_{a, c}=\varnothing$ otherwise.

Proof. If $[a, b, \Psi(c)] \neq \varnothing$, then $[a, b, \Psi(c)] \in T_{a, b} \cap T_{a, c}$, as $[a, b, \Psi(c)]=[a, c, \Psi(b)]$. Suppose $[a, b, x] \in T_{a, b} \cap T_{a, c}$, where $x \in \mathcal{C}$. Lemma 3.1 implies that $\{a, c\} \in\{\{a, \Psi(x)\}$, $\{b, a\},\{x, \Psi(b)\}\}$. Since $b \notin\{c, \Psi(c)\}$, either $\Psi(x)=c$ or $(a, c)=(\Psi(b), x)$ holds. In the former case, we have $[a, b, x]=[a, b, \Psi(c)]$. In the latter case, we also have $[a, b, x]=[\Psi(b), \Psi(a), \Psi(x)]=[a, b, \Psi(c)]$. Thus $T_{a, b} \cap T_{a, c}=\{[a, b, \Psi(c)]\}$ provided that $[a, b, \Psi(c)] \neq \varnothing$, and $T_{a, b} \cap T_{a, c}=\varnothing$ if $[a, b, \Psi(c)]=\varnothing$.

Lemma 3.4. Let $\{a, b\},\{c, d\}$ be distinct 2-sets of $\mathcal{C}$ and suppose $\Psi(w)=w$ for each $w \in\{a, b, c, d\}$. Then $T_{e, e} \cap T_{a, b} \cap T_{c, d}=T_{e, \Psi(e)} \cap T_{a, b} \cap T_{c, d}=\varnothing$ holds for any $e \in \mathcal{C}$.

Proof. If $e \neq \Psi(e)$, then we have $\{e, \Psi(e)\} \cap\{a, b\}=\varnothing$, and the assertion follows from Lemma 3.2. Hence we suppose that $e=\Psi(e)$.

Assume $T_{e, e} \cap T_{a, b} \cap T_{c, d} \neq \varnothing$. As $T_{a, b} \cap T_{c, d} \neq \varnothing$, Lemma 3.2 implies that $\{a, b\} \cap$ $\{c, d\} \neq \varnothing$. Thus we may assume $a=c$, and so $b \neq d$, as $\{a, b\} \neq\{c, d\}$. Lemma 3.3 implies $T_{a, b} \cap T_{a, d}=\{[a, b, d]\}$.

By Lemma 3.2, we can see that $T_{e, e} \cap T_{a, b} \neq \varnothing$ implies $\{e\} \cap\{a, b\} \neq \varnothing$, and that $T_{e, e} \cap T_{a, d} \neq \varnothing$ implies $\{e\} \cap\{a, d\} \neq \varnothing$. Since $b \neq d$, we have $a=e$, and $[a, b, d] \in T_{a, a}$. Lemma 3.1 implies $\{a\} \in\{\{a, d\},\{b, a\},\{d, b\}\}$. Hence, either $d=a$ or $b=a$, a contradiction.

Lemma 3.5. Suppose $a \in \mathcal{C}, a \neq \Psi(a)$, and $b, c, d, e \in \mathcal{C} \backslash\{a, \Psi(a)\}$. If $[a, b, c]=$ $[a, d, e] \neq \varnothing$, then $b=d$ and $c=e$.

Proof. Since $[a, b, c]=[a, d, e] \in T_{a, d}$, Lemma 3.1 implies that

$$
\{\Psi(a), d\} \in\{\{a, \Psi(c)\},\{b, \Psi(a)\},\{c, \Psi(b)\}\} .
$$

By assumption, we have $\{\Psi(a), d\}=\{b, \Psi(a)\}$, and $b=d$. Similarly, $c=e$ is obtained.

For $\mathcal{D} \subset \mathcal{C}$, we define $f(\mathcal{D})$ by $f(\mathcal{D})=2+2 \sum_{d \in \mathcal{D}} \delta_{d}$, where $\delta_{d}$ is defined as in (R1). If $\mathcal{D}=\Psi(\mathcal{D})$, then we define $\Delta(\mathcal{D})$ by $\Delta(\mathcal{D})=\{[x, y, z] \mid x, y, z \in \mathcal{C} \backslash \mathcal{D},[x, y, z] \neq \varnothing\}$.

Lemma 3.6. Let $\mathcal{D} \subset \mathcal{C}$. If $f(\mathcal{D})<|\Omega|$, then for any $a \in \mathcal{C}$ there exist $b, c \in \mathcal{C} \backslash \mathcal{D}$ such that $[a, b, \Psi(c)] \neq \varnothing$. Moreover, if $\mathcal{D}=\Psi(\mathcal{D})$, then $\Delta(\mathcal{D}) \neq \varnothing$.

Proof. Let $\alpha, \beta \in \Omega$ with $\phi(\alpha, \beta)=a$. Then

$$
\begin{aligned}
& \mid\{\omega \in \Omega \backslash\{\alpha, \beta\} \mid \phi(\alpha, \omega) \in \mathcal{D} \text { or } \phi(\beta, \omega) \in \mathcal{D}\} \mid \\
\leq & |\{\omega \in \Omega \backslash\{\alpha, \beta\} \mid \phi(\alpha, \omega) \in \mathcal{D}\}|+|\{\omega \in \Omega \backslash\{\alpha, \beta\} \mid \phi(\beta, \omega) \in \mathcal{D}\}| \\
\leq & 2 \sum_{d \in \mathcal{D}} \delta_{d} \\
< & |\Omega|-2=|\Omega \backslash\{\alpha, \beta\}| .
\end{aligned}
$$

Thus there exists $\gamma \in \Omega \backslash\{\alpha, \beta\}$ such that $\phi(\alpha, \gamma) \notin \mathcal{D}$ and $\phi(\beta, \gamma) \notin \mathcal{D}$. Setting $b=\phi(\beta, \gamma)$ and $c=\phi(\alpha, \gamma)$, we obtain $\{\alpha, \beta, \gamma\} \in[a, b, \Psi(c)]$ with $b, c \in \mathcal{C} \backslash \mathcal{D}$.

Suppose $\mathcal{D}=\Psi(\mathcal{D})$, and let $x \in \mathcal{C} \backslash \mathcal{D}$. By the first part of the lemma, there exist $y, z \in \mathcal{C} \backslash \mathcal{D}$ such that $[x, y, \Psi(z)] \neq \varnothing$. Since $\Psi(\mathcal{D})=\mathcal{D}$, we have $\Psi(z) \notin \mathcal{D}$, and hence $[x, y, \Psi(z)] \in \Delta(\mathcal{D})$. 


\section{Lower bounds of $\left|\overline{\Omega_{3}}\right|$}

This section is devoted to establishing some lower bounds for $\left|\overline{\Omega_{3}}\right|$, which will be needed later. As in the previous section, suppose that $(\Omega, \mathcal{C}, \phi, \Psi)$ is a regular edge-coloring. We define subsets $\mathcal{K}, \mathcal{L}, \mathcal{M}, \mathcal{K}_{1}, \mathcal{K}_{2}, \mathcal{L}_{1}, \mathcal{L}_{2}$ of $\mathcal{C}$ by

$$
\begin{aligned}
& \mathcal{K}=\{a \in \mathcal{C} \mid \Psi(a) \neq a\}, \mathcal{L}=\{a \in \mathcal{C} \mid \Psi(a)=a\}, \mathcal{M}=\left\{a \in \mathcal{C} \mid \delta_{a} \geq 2\right\} \\
& \mathcal{K}_{1}=\mathcal{K} \backslash \mathcal{M}, \mathcal{K}_{2}=\mathcal{K} \cap \mathcal{M}, \mathcal{L}_{1}=\mathcal{L} \backslash \mathcal{M}, \mathcal{L}_{2}=\mathcal{L} \cap \mathcal{M},
\end{aligned}
$$

and we define integers $k, l, m$ by

$$
k=\frac{|\mathcal{K}|}{2}, l=|\mathcal{L}|, m=|\mathcal{M}| .
$$

Lemma 4.1. Let $\left\{a_{1}, b_{1}\right\}, \ldots,\left\{a_{s}, b_{s}\right\}$ be distinct subsets of $\mathcal{C}$ with $T_{\Psi\left(a_{i}\right), b_{i}} \neq \varnothing$, for all $i$. Let $X=\bigcup_{1 \leq i \leq s} T_{\Psi\left(a_{i}\right), b_{i}}$. Then $|X| \geq\left\lceil\frac{s}{3}\right\rceil$. In particular

$$
\left|\overline{\Omega_{3}}\right| \geq\left\lceil\frac{2 m+|\mathcal{C}|(|\mathcal{C}|-1)}{6}\right\rceil .
$$

Proof. Since $T_{\Psi\left(a_{i}\right), b_{i}} \neq \varnothing$, we have

$$
\begin{aligned}
s & \leq\left|\left\{([x, y, z], j) \mid x, y, z \in \mathcal{C}, 1 \leq j \leq s,[x, y, z] \in T_{\Psi\left(a_{j}\right), b_{j}}\right\}\right| \\
& =\sum_{[x, y, z] \in \overline{\Omega_{3}}}\left|\left\{j \mid 1 \leq j \leq s,[x, y, z] \in T_{\Psi\left(a_{j}\right), b_{j}}\right\}\right| \\
& =\sum_{[x, y, z] \in X}\left|\left\{j \mid 1 \leq j \leq s,[x, y, z] \in T_{\Psi\left(a_{j}\right), b_{j}}\right\}\right| \\
& \leq \sum_{[x, y, z] \in X} 3 \\
& =3|X| .
\end{aligned}
$$

Since we can take $m+\left|\mathcal{C}_{(2)}\right|$ subsets $\left\{a_{i}, b_{i}\right\}$ with $T_{\Psi\left(a_{i}\right), b_{i}} \neq \varnothing$, the second part follows.

Lemma 4.2. For any $a \in \mathcal{K}_{2}$, the inequality $\left|U_{a} \cup V_{a}\right| \geq 2$ holds.

Proof. Let $\alpha, \beta \in \Omega$ be such that $\phi(\alpha, \beta)=a$. There exists $\gamma \in \Omega \backslash\{\alpha, \beta\}$ such that $\phi(\beta, \gamma)=a$ and $\phi(\alpha, \gamma) \neq a$, as

$$
|\{\omega \in \Omega \backslash\{\beta\} \mid \phi(\alpha, \omega)=a\}|=\delta_{a}-1<\delta_{a}=|\{\omega \in \Omega \mid \phi(\beta, \omega)=a\}| .
$$

We have $[\{\alpha, \beta, \gamma\}]=[a, a, \phi(\gamma, \alpha)] \in V_{a}$. If $\left|U_{a} \cup V_{a}\right|=1$, then $U_{a}=V_{a}$, and $[a, a, \phi(\gamma, \alpha)] \in U_{a}$. Lemma 3.1 implies

$$
\{a\} \in\{\{a,(\Psi \circ \phi)(\gamma, \alpha)\},\{a, \Psi(a)\},\{\phi(\gamma, \alpha), \Psi(a)\}\},
$$

which is a contradiction.

Lemma 4.3. $\left|\overline{\Omega_{3}}\right| \geq \max \left\{m,\left\lceil\frac{4 m+l(l-1)}{6}\right\rceil\right\}$. 
Proof. Let $\mathcal{K}^{\prime}$ denote a subset of $\mathcal{K}$ such that $\mathcal{K}=\mathcal{K}^{\prime} \cup \Psi\left(\mathcal{K}^{\prime}\right)$ and $\mathcal{K}^{\prime} \cap \Psi\left(\mathcal{K}^{\prime}\right)=\varnothing$. Let $\Gamma=\left(\bigcup_{a \in \mathcal{K}^{\prime} \cap \mathcal{M}} U_{a} \cup V_{a}\right) \cup\left(\bigcup_{b \in \mathcal{L}_{2}} U_{b}\right)$. Lemma 3.2 implies that $|\Gamma|=\sum_{a \in \mathcal{K}^{\prime} \cap \mathcal{M}} \mid U_{a} \cup$ $V_{a}\left|+\sum_{b \in \mathcal{L}_{2}}\right| U_{b} \mid$. By Lemma 4.2, we have that $|\Gamma| \geq 2\left|\mathcal{K}^{\prime} \cap \mathcal{M}\right|+\left|\mathcal{L}_{2}\right|=m$. In particular, $\left|\overline{\Omega_{3}}\right| \geq m$.

We define $X=\left\{\{c, d\} \in \mathcal{L}_{(2)} \mid T_{c, d} \cap \Gamma \neq \varnothing\right\}$. We claim $|X| \leq|\Gamma|$. Indeed, if $|X|>$ $|\Gamma|$, then there exist $\{c, d\},\left\{c^{\prime}, d^{\prime}\right\} \in X$ such that $\Gamma \cap T_{c, d} \cap T_{c^{\prime}, d^{\prime}} \neq \varnothing$. By the definition of $\Gamma$, this contradicts Lemma 3.4, and the claim holds. Equivalently, $\left|\mathcal{L}_{(2)} \backslash X\right| \geq\left(\begin{array}{l}l \\ 2\end{array}\right)-|\Gamma|$. Now, Lemma 4.1 implies that

$$
\left|\overline{\Omega_{3}}\right| \geq\left|\Gamma \cup \underset{\{c, d\} \in \mathcal{L}_{(2)} \backslash X}{ } T_{c, d}\right|=|\Gamma|+\left|\bigcup_{\{c, d\} \in \mathcal{L}_{(2)} \backslash X} T_{c, d}\right| \geq\left\lceil\frac{4 m+l(l-1)}{6}\right\rceil .
$$

Lemma 4.4. Let $\mathcal{D} \subset \mathcal{C}$. If $\mathcal{D}=\Psi(\mathcal{D})$, then

$$
\left|\overline{\Omega_{3}}\right| \geq|\Delta(\mathcal{D})|+|\{[a, x, y] \mid a \in \mathcal{D}, x, y \in \mathcal{C} \backslash \mathcal{D},[a, x, y] \neq \varnothing\}|+\left|\bigcup_{b, c \in \mathcal{D}} T_{b, c}\right| .
$$

Proof. Lemma 3.1 implies that the sets corresponding to the terms of the right-hand side are disjoint. Hence the claim holds.

Lemma 4.5. Suppose $\mathcal{D} \subset \mathcal{C}, \mathcal{D}=\Psi(\mathcal{D})$ and $f(\mathcal{D})<|\Omega|$. Then $\left|\overline{\Omega_{3}}\right| \geq 1+|\mathcal{D}|+$ $\left|\bigcup_{b, c \in \mathcal{D}} T_{b, c}\right|$.

Proof. By assumption, Lemma 3.6 implies that $\Delta(\mathcal{D}) \neq \varnothing$. Also, for each $a \in \mathcal{D}$, Lemma 3.6 implies that there exist $x_{a}, y_{a} \in \mathcal{C} \backslash \mathcal{D}$ such that $\left[a, x_{a}, \Psi\left(y_{a}\right)\right] \neq \varnothing$. We have $x_{a} \in \mathcal{C} \backslash \mathcal{D}$ and $\Psi\left(y_{a}\right) \in \mathcal{C} \backslash \mathcal{D}$, as $\Psi(\mathcal{D})=\mathcal{D}$. Lemma 4.4 implies that

$$
\left|\overline{\Omega_{3}}\right| \geq|\Delta(\mathcal{D})|+\sum_{a \in \mathcal{D}}\left|\left\{\left[a, x_{a}, \Psi\left(y_{a}\right)\right]\right\}\right|+\left|\bigcup_{b, c \in \mathcal{D}} T_{b, c}\right| \geq 1+|\mathcal{D}|+\left|\bigcup_{b, c \in \mathcal{D}} T_{b, c}\right| .
$$

\section{Proof of the main result}

In this section we prove Theorem 1.2. Let a regular edge-coloring $(\Omega, \mathcal{C}, \phi, \Psi)$ be given. Let $U_{c}, V_{c}, \mathcal{K}, \mathcal{L}, \mathcal{M}, k, l, m$ be as in (2.1), (4.1) and (4.2).

Lemma 5.1. Assume $|\Omega| \geq 8$. If $m=0$ or $|\mathcal{C}| \geq 6$, then $\left|\overline{\Omega_{3}}\right| \geq|\mathcal{C}|$ holds.

Proof. If $m=0$, then $|\mathcal{C}|=|\Omega|-1>6$. If $|\mathcal{C}|>6$, then Lemma 4.1 implies $\left|\overline{\Omega_{3}}\right| \geq|\mathcal{C}|$. If $|\mathcal{C}|=6$, then $m \geq 1$, as $|\Omega| \geq 8$. Therefore the result follows from Lemma 4.1.

Lemma 5.2. If $|\Omega| \geq 11$ and $\mathcal{K}_{1} \neq \varnothing$, then $\left|\overline{\Omega_{3}}\right| \geq|\mathcal{C}|$.

Proof. Let $c \in \mathcal{K}_{1}$. The condition (R1) implies that there exist $\alpha_{1}, \alpha_{2}, \alpha_{3} \in \Omega$ such that $\phi\left(\alpha_{1}, \alpha_{2}\right)=\phi\left(\alpha_{2}, \alpha_{3}\right)=c$. We define $d=\phi\left(\alpha_{3}, \alpha_{1}\right), \mathcal{D}=\{c, \Psi(c)\}$, and $\mathcal{E}=\mathcal{C} \backslash \mathcal{D}$. Since $|\Omega| \geq 11$ and $\delta_{c}=1$, we get that $\mathcal{E} \neq \varnothing$, and that

$$
\text { for all } \omega \in \Omega \backslash\left\{\alpha_{1}, \alpha_{3}\right\}, \phi\left(\omega, \alpha_{2}\right) \in \mathcal{E} .
$$

By (R1), for any $e \in \mathcal{E} \backslash\{d\}$, there exists $\beta_{e} \in \Omega \backslash\left\{\alpha_{1}\right\}$ such that $\phi\left(\alpha_{3}, \beta_{e}\right)=e$. We have $\phi\left(\beta_{e}, \alpha_{2}\right) \in \mathcal{E}$ by (5.1). It follows that

$$
|\{(x, y) \mid x \in \mathcal{E} \backslash\{d\}, y \in \mathcal{E},[c, x, y] \neq \varnothing\}| \geq|\mathcal{E} \backslash\{d\}| .
$$


As $f(\mathcal{D})=6<|\Omega|$, Lemma 3.6 implies $\Delta(\mathcal{D}) \neq \varnothing$. Lemma 4.4 implies that

$$
\begin{aligned}
\left|\overline{\Omega_{3}}\right| \geq|\Delta(\mathcal{D})|+|\{[a, x, y] \mid a \in \mathcal{D}, x, y \in \mathcal{E},[a, x, y] \neq \varnothing\}|+\left|\bigcup_{b, b^{\prime} \in \mathcal{D}} T_{b, b^{\prime}}\right| \\
=|\Delta(\mathcal{D})|+|\{[c, x, y] \mid x, y \in \mathcal{E},[c, x, y] \neq \varnothing\}|+\left|V_{c}\right| \\
=|\Delta(\mathcal{D})|+|\{(x, y) \mid x, y \in \mathcal{E},[c, x, y] \neq \varnothing\}|+\left|V_{c}\right| \\
=|\Delta(\mathcal{D})|+|\{(x, y) \mid x \in \mathcal{E} \backslash\{d\}, y \in \mathcal{E},[c, x, y] \neq \varnothing\}| \\
\quad+|\{y \mid y \in \mathcal{E},[c, d, y] \neq \varnothing\}|+\left|V_{c}\right| .
\end{aligned}
$$

Combining the inequalities $|\Delta(\mathcal{D})| \geq 1,\left|V_{c}\right| \geq 1$, and (5.2), we obtain

$$
\left|\overline{\Omega_{3}}\right| \geq 1+|\mathcal{C}|-|\{c, \Psi(c), d\}|+0+1 \geq|\mathcal{C}|-1 \text {. }
$$

Suppose $\left|\overline{\Omega_{3}}\right|<|\mathcal{C}|$. Then equality is forced in each of the above inequalities. In particular, $|\Delta(\mathcal{D})|=1, d \notin\{c, \Psi(c)\}$, and $[c, d, y]=\varnothing$ for any $y \in \mathcal{E}$.

Now, if there exists $\gamma \in \Omega \backslash\left\{\alpha_{1}\right\}$ such that $\phi\left(\alpha_{3}, \gamma\right)=d$, then (5.1) implies $\phi\left(\gamma, \alpha_{2}\right) \in$ $\mathcal{E}$. This would imply $[c, d, y] \neq \varnothing$, where $y=\phi\left(\gamma, \alpha_{2}\right) \in \mathcal{E}$, which is a contradiction. Thus there is no such $\gamma$. That is, $\delta_{d}=1$. Hence $f(\mathcal{D} \cup\{d, \Psi(d)\}) \leq 10<|\Omega|$, and Lemma 3.6 implies $\Delta(\mathcal{D} \cup\{d, \Psi(d)\}) \neq \varnothing$. We have $\Delta(\mathcal{D})=\Delta(\mathcal{D} \cup\{d, \Psi(d)\})$, as $|\Delta(\mathcal{D})|=1$. However, since $f(\{c, \Psi(c)\})=6<|\Omega|$, Lemma 3.6 implies that there exist $z, w \in \mathcal{E}$ such that $[d, z, w] \neq \varnothing$. Since $[d, z, w] \in \Delta(\mathcal{D})$ and $[d, z, w] \notin \Delta(\mathcal{D} \cup\{d, \Psi(d)\})$, we have $\Delta(\mathcal{D}) \neq \Delta(\mathcal{D} \cup\{d, \Psi(d)\})$, which is a contradiction. Therefore $\left|\overline{\Omega_{3}}\right| \geq|\mathcal{C}|$.

Lemma 5.3. If $|\Omega| \geq 11$ and $\mathcal{L}=\varnothing$, then $\left|\overline{\Omega_{3}}\right| \geq|\mathcal{C}|$.

Proof. By assumption, we have $\mathcal{C}=\mathcal{K}_{1} \cup \mathcal{M}$. If $\mathcal{K}_{1}=\varnothing$, then the result follows from Lemma 4.3. Otherwise the result follows from Lemma 5.2.

Lemma 5.4. If $|\Omega| \geq 11$ and $\mathcal{K}=\varnothing$, then $\left|\overline{\Omega_{3}}\right| \geq|\mathcal{C}|$.

Proof. By Lemma 5.1, we may assume $l \leq 5$ and $m \geq 1$. If $m \geq 2$, then Lemma 4.3 yields the result. Assume $m=1$. Set $\mathcal{C}=\{1, \ldots, l\}$. Then we may assume without loss of generality that $\delta_{1} \geq 2$. Since $f(\mathcal{C} \backslash\{1\})=2 l<|\Omega|$, Lemma 3.6 yields $[c, 1,1] \neq \varnothing$ for each $c \in \mathcal{C}$. Therefore $\left|\overline{\Omega_{3}}\right| \geq l$.

Lemma 5.5. Let $(\Omega, \mathcal{C}, \phi, \Psi)$ be a regular edge-coloring with $|\Omega| \geq 11$ and $|\mathcal{C}|=3$. Then $\left|\overline{\Omega_{3}}\right| \geq 3$ holds.

Proof. We argue by contradiction. Assume $\left|\overline{\Omega_{3}}\right|<3$. Lemmas 5.3 and 5.4 imply $\mathcal{C}=$ $\{1, \overrightarrow{1}, \overleftarrow{1}\}$, where $\Psi(1)=1$ and $\Psi(\overrightarrow{1})=\overleftarrow{1}$. Also, $\left|\overline{\Omega_{3}}\right|<|\mathcal{C}|$ implies $\delta_{\overrightarrow{1}} \geq 2$ by Lemma 5.2. We have $\delta_{1}=1$ by Lemma 4.3. Since $\left|U_{\overrightarrow{1}} \cup V_{\overrightarrow{1}}\right| \geq 2$ by Lemma 4.2, it follows from $\left|\overline{\Omega_{3}}\right| \leq 2$ that

$$
\overline{\Omega_{3}}=U_{\overrightarrow{1}} \cup V_{\overrightarrow{1}} .
$$

Now, since $[1, \overrightarrow{1}, \overleftarrow{1}] \notin U_{\overrightarrow{1}} \cup V_{\overrightarrow{1}}$ by Lemma 3.1 , we have $[1, \overrightarrow{1}, \overleftarrow{1}]=\varnothing$, which implies $T_{1, \overrightarrow{1}}=\{[1, \overrightarrow{1}, \overrightarrow{1}]\}$ by (5.3). Since $f(\{1\})=4<|\Omega|$, Lemma 3.6 implies $\Delta(\{1\}) \neq \varnothing$. Hence

$$
\overline{\Omega_{3}}=\{[1, \overrightarrow{1}, \overrightarrow{1}]\} \cup \Delta(\{1\}) .
$$


We compare (5.3) with (5.4). Since $[1, \overrightarrow{1}, \overrightarrow{1}] \notin U_{\overrightarrow{1}}$ by Lemma 3.2, we have $U_{\overrightarrow{1}}=$ $\Delta(\{1\})$, which implies $U_{\overrightarrow{1}}=\{[\overrightarrow{1}, \overrightarrow{1}, \overleftarrow{1}]\}$. Hence $\overline{\Omega_{3}}=\{[1, \overrightarrow{1}, \overrightarrow{1}],[\overrightarrow{1}, \overrightarrow{1}, \overleftarrow{1}]\}$.

Let $\alpha_{1} \in \Omega$. Pick distinct $\beta_{1}, \alpha_{2}, \beta_{2}, \alpha_{3} \in \Omega$ in such a way that $\phi\left(\alpha_{1}, \beta_{1}\right)=1$, $\phi\left(\alpha_{1}, \alpha_{2}\right)=\overrightarrow{1}, \phi\left(\alpha_{2}, \beta_{2}\right)=1$, and $\phi\left(\alpha_{2}, \alpha_{3}\right)=\overrightarrow{1}$. Notice that $\delta_{1}=1$. Since $\phi\left(\alpha_{1}, \alpha_{2}\right)=\phi\left(\alpha_{2}, \alpha_{3}\right)=\overrightarrow{1}$, we have $\phi\left(\alpha_{1}, \alpha_{3}\right)=\overrightarrow{1}$. Since $\phi\left(\alpha_{1}, \alpha_{2}\right)=\overrightarrow{1}$ and $\phi\left(\alpha_{2}, \beta_{2}\right)=1$, we have $\phi\left(\beta_{2}, \alpha_{1}\right)=\overrightarrow{1}$. Similarly, we have $\phi\left(\alpha_{3}, \beta_{2}\right)=\overrightarrow{1}$. Thus, $\left[\left\{\alpha_{1}, \alpha_{3}, \beta_{2}\right\}\right]=[\overrightarrow{1}, \overrightarrow{1}, \overrightarrow{1}]$, a contradiction.

We are now ready to prove our main result.

Theorem 5.6. Let $(\Omega, \mathcal{C}, \phi, \Psi)$ be a regular edge-coloring. If $|\Omega| \geq 11$, then $\left|\overline{\Omega_{3}}\right| \geq|\mathcal{C}|$.

Proof. Let $\mathcal{K}=\{\overrightarrow{1}, \ldots, \vec{k}, \overleftarrow{1}, \ldots, \overleftarrow{k}\}, \mathcal{L}=\{1, \ldots, l\}$, and $\Psi(\vec{i})=\overleftarrow{i}$. By Lemmas 5.1, 5.3, 5.4, and 5.5, we only need to consider the cases where $(k, l,|\mathcal{C}|)=(1,2,4)$, $(1,3,5)$, and $(2,1,5)$. In each case, we may assume $\delta_{\vec{i}} \geq 2$ for $1 \leq i \leq k$, by Lemma 5.2. Lemma 4.2 implies that

$$
\left|U_{\vec{i}} \cup V_{\vec{i}}\right| \geq 2 . \quad(1 \leq i \leq k)
$$

Also, Lemma 3.2 implies that

$$
\left(U_{\vec{i}} \cup V_{\vec{i}}\right) \cap T_{j, j^{\prime}}=\varnothing . \quad\left(1 \leq i \leq k, 1 \leq j, j^{\prime} \leq l\right)
$$

Notice that $T_{a, b} \neq \varnothing$ unless $b=\Psi(a)$ and $\delta_{a}=1$, for any $a, b \in \mathcal{C}$.

Suppose $(k, l)=(1,2)$. If $\delta_{1}=\delta_{2}=1$, then Lemma 4.5 implies $\left|\overline{\Omega_{3}}\right| \geq 4$, so we may assume without loss of generality that $\delta_{1} \geq 2$. If $\left|U_{1} \cup T_{1,2}\right| \geq 2$, then $\left|\overline{\Omega_{3}}\right| \geq \mid U_{\overrightarrow{1}} \cup V_{\overrightarrow{1}} \cup$ $U_{1} \cup T_{1,2}|=| U_{\overrightarrow{1}} \cup V_{\overrightarrow{1}}|+| U_{1} \cup T_{1,2} \mid \geq 4$ by (5.5) and (5.6). If $\left|U_{1} \cup T_{1,2}\right|=1$, then $U_{1}=T_{1,2}$, and $U_{1} \cup T_{1,2}=\{[1,1,2]\}$ by Lemma 3.3. In particular, we get $[1,1,2] \neq \varnothing$. Lemmas 3.1 and 3.2 imply that $\{[1,1,2]\}, U_{\overrightarrow{1}}, T_{1, \overrightarrow{1}}$, and $T_{2}, \overrightarrow{1}$ are pairwise disjoint. Hence we obtain $\left|\overline{\Omega_{3}}\right| \geq 4$ by collecting these families.

Next suppose $(k, l)=(1,3)$. If $[1,2,3]=\varnothing$, then Lemma 3.3 implies that $T_{1,2}$, $T_{1,3}$, and $T_{2,3}$ are pairwise disjoint. Hence, $\left|\overline{\Omega_{3}}\right| \geq\left|U_{\overrightarrow{1}} \cup V_{\overrightarrow{1}} \cup T_{1,2} \cup T_{1,3} \cup T_{2,3}\right| \geq 5$ by (5.5) and (5.6). We may assume that $[1,2,3] \neq \varnothing$. Lemmas 3.1 and 3.2 imply that $\{[1,2,3]\}, U_{\overrightarrow{1}}, T_{1, \overrightarrow{1}}, T_{2, \overrightarrow{1}}$, and $T_{3, \overrightarrow{1}}$ are pairwise disjoint. Collecting these families, we have $\left|\overline{\Omega_{3}}\right| \geq 5$.

Finally suppose $(k, l)=(2,1)$. Assume $\left|T_{1, \overrightarrow{1}} \cup T_{1, \overrightarrow{2}}\right| \geq 2$. Lemma 3.2 implies that $\left(T_{1, \overrightarrow{1}} \cup T_{1, \overrightarrow{2}}\right), T_{\Psi(\overrightarrow{1}), \overrightarrow{2}}, U_{\overrightarrow{1}}$, and $U_{\overrightarrow{2}}$ are pairwise disjoint. Hence $\left|\overline{\Omega_{3}}\right| \geq \mid T_{1, \overrightarrow{1}} \cup$ $T_{1, \overrightarrow{2}} \cup T_{\Psi(\overrightarrow{1}), \overrightarrow{2}} \cup U_{\overrightarrow{1}} \cup U_{\overrightarrow{2}} \mid \geq 5$. We may assume that $\left|T_{1, \overrightarrow{1}} \cup T_{1, \overrightarrow{2}}\right|=1$. Then $T_{1, \overrightarrow{1}}=T_{1, \overrightarrow{2}}$, and $T_{1, \overrightarrow{1}} \cup T_{1, \overrightarrow{2}}=\{[1, \overrightarrow{1}, \overleftarrow{2}]\}$ by Lemma 3.3. In particular, we get $[1, \overrightarrow{1}, \overleftarrow{2}] \neq \varnothing$. Lemma 3.2 implies that $\{[1, \overrightarrow{1}, \overleftarrow{2}]\}, U_{\overrightarrow{1}} \cup V_{\overrightarrow{1}}$, and $U_{\overrightarrow{2}} \cup V_{\overrightarrow{2}}$ are pairwise disjoint. Therefore $\left|\overline{\Omega_{3}}\right| \geq\left|\{[1, \overrightarrow{1}, \overleftarrow{2}]\} \cup U_{\overrightarrow{1}} \cup V_{\overrightarrow{1}} \cup U_{\overrightarrow{2}} \cup V_{\overrightarrow{2}}\right| \geq 5$ by (5.5).

Proof of Theorem 1.2. Let $\left(\Omega, \mathcal{C}_{G}, \phi_{G}, \Psi_{G}\right)$ be the regular edge-coloring induced by $G$. By the definition of induced regular edge-coloring, $\left|\mathcal{C}_{G}\right|=\left|\Omega_{[2]} / G\right|$ holds. For $A, B \in \Omega_{(3)}$, if $A^{g}=B$ for some $g \in G$, then $[A]=[B]$, hence $\left|\overline{\Omega_{3}}\right| \leq\left|\Omega_{(3)} / G\right|$ holds. The result follows from Theorem 5.6. 


\section{Acknowledgements}

The author would like to thank his supervisor Akihiro Munemasa for valuable suggestions and comments. The author would also like to thank the anonymous referees for pointing out mistakes in an earlier version of this paper, and for their valuable suggestions.

\section{References}

[1] P. J. Cameron and J. Saxl, Permuting unordered subsets, Quart. J. Math. Oxford Ser. (2) 34 (1983), no. 134, 167-170.

[2] J. D. Dixon and B. Mortimer, Permutation Groups, Springer-Verlag, New York, 1996.

[3] D. Livingstone and A. Wagner, Transitivity of finite permutation groups on unordered sets, Math. Zeitschr. 90 (1965), 393-403.

[4] W. J. Martin and B. E. Sagan, A new notion of transitivity for groups and sets of permutations, J. London Math. Soc. (2) 73 (2006), 1-13.

[5] B. E. Sagan, The Symmetric Group, second ed., Springer-Verlag, New York, 2001. 